\title{
Substrate Integrated Waveguide Textile Antennas as Energy Harvesting Platforms
}

\author{
Sam Lemey and Hendrik Rogier \\ Department of Information Technology \\ Ghent University/iMinds \\ Ghent, Belgium \\ Sam.Lemey@intec.ugent.be
}

\begin{abstract}
Textile multi-antenna systems are key components of smart fabric and interactive textile (SFIT) systems, as they establish reliable and energy-efficient wireless body-centric communication links. In this work, we investigate how their functionality can further be extended by exploiting their surface as an energy harvesting and power management platform. We provide guidelines for selecting an appropriate antenna topology and describe a suitable integration procedure. We demonstrate this approach by integrating two flexible solar cells, a microenergy cell and a flexible power management system onto a well-chosen wearable substrate integrated waveguide cavitybacked textile slot antenna, without affecting its performance, to enable energy harvesting from solar and artificial light. In addition, the compact and highly-integrated harvesting module provides a terminal for connecting a thermoelectric generator, enabling thermal body energy harvesting. Measurements in a realistic indoor environment have demonstrated that this hybrid energy harvesting approach leverages a more continuous flow of scavenged energy, enabling energy scavenging in most of the indoor and outdoor scenarios.
\end{abstract}

\section{INTRODUCTION}

Recently, a lot of research focused on increasing the operational safety and efficiency of police, fire fighter and rescue interventions. In this regard, several wearable electronic systems, unobtrusively integrated into the garment [1], were designed to enable remote monitoring of vital signs, activities and environmental parameters by wirelessly relaying these data to a remote location for supervision. However, a frequently heard complaint by the end users of such a smart fabric and interactive textile (SFIT) systems concerns the limited system autonomy [2]. To increase system autonomy without using heavy batteries, lots of research efforts were devoted to increase the efficiency of the electronic circuitry. As [3] indicates that the wireless communication subsystem is one of the major power consumers, on the one hand, textile antennas should preferable exhibit a large radiation efficiency and high gain, in order to reduce power consumption. Moreover, multiple highly-efficient textile antennas could be deployed onto the human body to further reduce power consumption [4]. On the other hand, many physical quantities that are monitored by sensors in smart textiles change slowly. Hence, they can be measured and transmitted in bursts, making use of a low duty cycle operation regime [5]. All these reasons point to SFIT systems as prime candidates to be partly, or even solely, powered by energy harvesting techniques [5]. By extracting energy from the user's activities [6], [7], or from ambient sources at the place where the SFIT is worn by the user [7], [8], life-threatening situations, due to batteries running out of power during interventions, could be prevented. Preferably, energy is scavenged from multiple different energy sources [5], [7] to increase the amount of harvested energy and to obtain a higher continuity in energy scavenging. Anyhow, a power management system is required to convert the small and highly fluctuating levels of energy into a useful form. Altough professional garments provide sufficient space to integrate a textile multi-antenna system, a smart approach is required for the integration of additional energy harvesting transducers, and the corresponding power management hardware, to maintain the user's comfort, on the one hand, and to scavenge sufficient energy, on the other hand, without affecting antenna or energy harvesting performance.

Therefore, in this work, we investigate the potential of reusing a textile antenna as an integration platform for energy harvesting and power management hardware. First, we select a suitable antenna topology, exhibiting a very high isolation from its environment, facilitating integration of additional hardware and enabling unobtrusive integration. Then, we describe the integration of a flexible hybrid energy scavenging system onto the textile antenna, leveraging energy harvesting from three different energy sources. In detail, a flexible central power management system (CPMS), including energy-storage, is placed on the antenna's backside. It is designed for energy harvesting from outdoor solar light and indoor artificial light by means of two different flexible solar cells, integrated on top of the textile antenna. In addition, an ultralow-voltage step-up converter is included to enable thermal body energy harvesting by means of a thermoelectric generator (TEG), placed on the human skin. The work, performed in [5], has been extended with a comprehensive field test in a realistic indoor environment in order to emphasize the importance of hybrid energy harvesting in realistic scenarios.

\section{SyStem OVERVIEW}

Fig. 1 illustrates the goal of this contribution, being the design of a compact, highly-integrated and unobtrusive module, in which the surface required by the textile antenna for efficient communication is reused as an energy harvesting and power management platform, able to combine the energy scavenged 


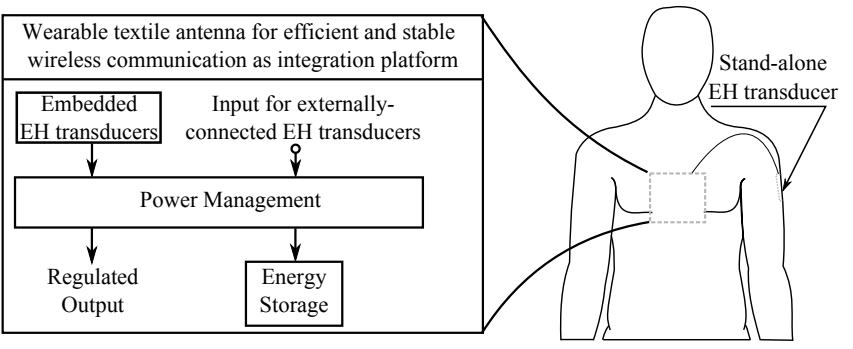

Fig. 1. System concept and application.

by multiple embedded and/or externally connected energy harvesting (EH) transducers, in order to provide a regulated voltage for adequately powering a wireless communication module. By carefully selecting diverse energy sources from which energy is extracted, such a hybrid energy harvesting approach leverages a higher total harvested energy and reduces the time, during which no energy can be harvested.

\section{A. SIW cavity-backed slot antenna as integration platform}

First, the selection of an appropriate antenna topology is of major importance in order to facilitate integration of additional hardware without degradation in radiation performance, in the meantime maintaining the user's comfort. We have selected the wearable substrate integrated waveguide (SIW) cavitybacked textile slot antenna, presented in [9], as the basis for our highly-integrated flexible hybrid energy harvesting textile antenna system. This wearable textile antenna is designed for operation in the $2.45 \mathrm{GHz}$ ISM-band and is depicted in Fig. 2. A cavity-backed slot antenna topology is adopted, because such a topology exhibits an excellent isolation from its environment, making it not only extremely suitable for on-body deployment, but also for the integration of additional electronic hardware. A second reason to adopt this topology is the ability to realize a flexible, low-profile and conformal design. This is demonstrated by the selected design, in which a closed cell expanded rubber protective foam, typically used as shock absorber in fire fighter jackets, is adopted as antenna substrate and the antenna's slot and feed layers are constructed using a copper-coated nylon fabric. Thermally-activated adhesive sheets are used to glue both conductive fabric layers to the antenna substrate. The SIW technology, in which eyelets are used to implement conductive walls, is adopted to implement the side walls of the antenna cavity (Fig. 2). The third reason to adopt this antenna topology concerns the ease by which additional hardware can be integrated. Details are given in Section II-B. The main dimensions of the selected antenna are depicted in Fig. 2, whereas more detailed information can be found in [9].

\section{B. Energy harvesting hardware}

The antenna surface is then reused as an energy harvesting and power management platform, by integrating a flexible power management module, a micro-energy cell and two offthe-shelf flexible solar cells. An overview of our system is depicted in Fig. 2. As can be seen, the flexible power management system consists of two interconnected subsystems,
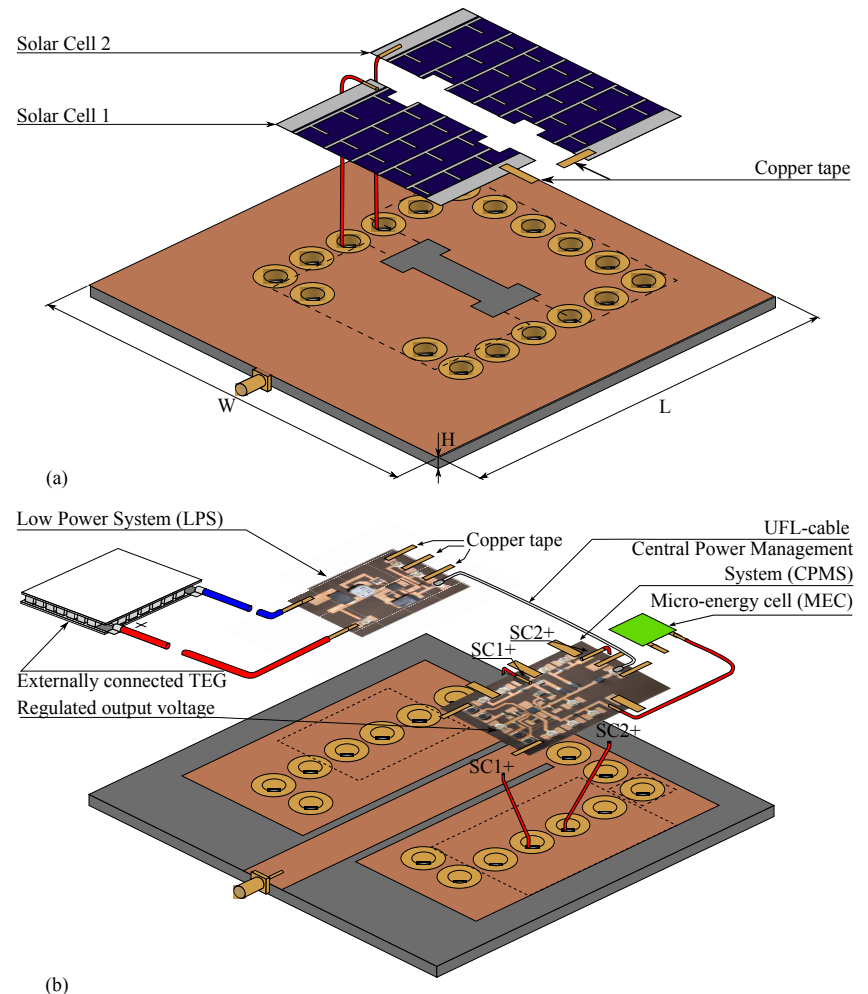

Fig. 2. The textile SIW cavity-backed slot antenna proposed in [9] $(\mathrm{W}=131.5$ $\mathrm{mm}, \mathrm{L}=126.5 \mathrm{~mm}$ and $\mathrm{H}=4 \mathrm{~mm}$ ), with two flexible a-Si:H solar cells glued on top, the proposed power management module and energy storage device, integrated directly underneath the textile antenna, and the externally connected TEG. (a) Slot plane; (b) Feed plane.

being the low power system (LPS) and the CPMS. The CPMS is built around the MAX17710 IC by Maxim Integrated. It is designed to charge the MEC from two high-voltage DC sources by means of linear harvesting and one low-voltage DC source via a boost converter. By connecting solar cell 1 to the boost converter input and solar cell 2 to a linear harvesting input, energy harvesting from artificial and solar light is enabled, respectively. The LPS is based on the LTC3108 IC by Linear Technology. It implements an ultralow-voltage stepup converter, of which the output is connected to a linear harvesting input of the CPMS. This enables thermal body energy harvesting via an externally connected TEG, based on Seebeck's effect [6], exploiting the thermal gradient between human skin and ambient temperature. The power available from these three different energy sources is combined by the CPMS to charge the micro-energy cell (MEC), in the meantime protecting the MEC and providing a regulated output voltage. As depicted in Fig. 2, the TEG was not integrated onto the antenna to obtain a higher degree of freedom. It allows the wearer to connect either a single TEG or multiple TEGs (potentially stacked), with or without heat sink. In this work, a single TEG ( the UltraTec Series UT6,24,F1,5555 from Laird Technologies), tightly attached with its hot side to the skin whereas its cold side is exposed to the ambient air, is connected.

Mechanical flexibility of the LPS and CPMS circuits is obtained by etching both circuits on a flexible, ultra-thin 
polyimide substrate and using small electronic components, distributed over a larger area than necessary, as in [8]. Both circuits are glued to the antenna's feed plane with stretchable adhesive sheets, leaving the feed line uncovered. At the antenna's feed plane, a $170 \mu m$-thick MEC (a MEC-225 from Thinergy) is glued on top of an eyelet, of approximately the same size, to maintain flexibility. Two ultra-flexible $200 \mu m$-thick PowerFilm thin film a-Si:H-solar cells are attached to the antenna's slot plane as depicted in Fig. 2, after patterning them according to the antenna's slot.

The amount and length of interconnecting wires is minimized by exploiting the antenna's properties. First of all, the antenna cavity acts as a DC ground, which allows us to use copper tape to connect the cathode of both solar cells, the MEC's negative side and the DC ground of both circuits to the SIW cavity in order to share a common ground. Moreover, employing hollow eyelets allows us to route the positive connection of both solar cells to the power management system without affecting the antenna's performance.

\section{System PERformance}

\section{A. Antenna performance}

The antenna performance was already extensively evaluated in [9] to demonstrate its excellent behavior with respect to on-body deployment and under bending. In this section, the influence of integrating energy harvesting and power management hardware on the antenna performance is investigated. Therefore, the antenna's radiation pattern at $2.45 \mathrm{GHz}$ and the antenna's return loss characteristic from 2.0 to $3.0 \mathrm{GHz}$ is measured in an anechoic room before integration of any energy harvesting hardware, after solar cell integration, and, finally, after circuitry integration. In case of the solar cell integration, the copper tape for connecting both solar cells cathodes to the antenna cavity and the wires for connecting the anodes of the solar cells to the power management module are included in the measurement. In the third measurement setup, also including the effect of the MEC and the circuitry, all required connections (as depicted in Fig. 2) are made and included in the measurement. The antenna gain in the E- and $\mathrm{H}$-plane at $2.45 \mathrm{GHz}$, for each measurement setup, is shown in Fig. 3, whereas Table I depicts the center frequency and the -10 dB-impedance bandwidth for all three measurement setups. Fig. 3 and Table I prove that the integration of the energy harvesting hardware only has a minimal influence on antenna performance and, hence, that the selected antenna topology is a very suitable antenna topology for the integration of additional energy harvesting and power management hardware.

\section{B. Energy harvesting performance in a realistic environment}

A male test person of average height, wearing the proposed system on his chest and the selected off-the-shelf TEG on his left arm (Fig. 1), is considered to demonstrate the energy harvesting potential of our proposed system in a realistic indoor and outdoor environment. First, the performance of our system is investigated in a realistic indoor environment by concatenating four well-chosen indoor scenarios, each
TABLE I

MEAsured CENTER FREQUENCY $f_{c}$ AND BANDWIDTH $B W$ OF THE SIW CAVITY-BACKED TEXTILE SLOT ANTENNA.

\begin{tabular}{ccc}
\hline \hline & $f_{c}[\mathrm{GHz}]$ & $B W[\mathrm{MHz}]$ \\
\hline \hline Antenna without additional hardware & 2.411 & 325 \\
Antenna with solar cells & 2.406 & 330 \\
Antenna with energy harvesting hardware & 2.408 & 396 \\
\hline
\end{tabular}
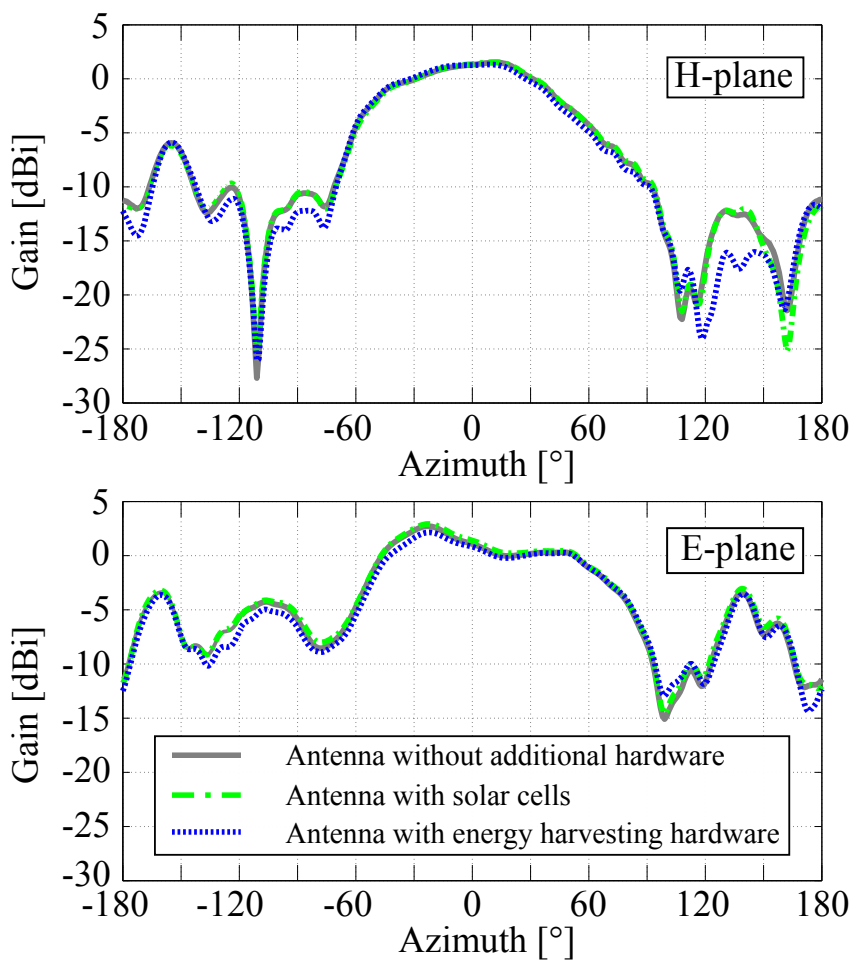

Fig. 3. Measured radiation pattern of the SIW cavity-backed slot antenna at $2450 \mathrm{MHz}$

lasting 20 minutes. Throughout the four scenarios, the power generated by each of the three energy harvesting transducers is monitored as well as the effectively stored energy in the MEC. Since measurements pointed out that solar cell 2 was not able to harvest energy in these indoor scenarios, only the powers generated by solar cell 1 and the TEG are depicted in Fig. 4. At the start of the measurement, the test person is sitting in a $2.4 \mathrm{~m}$ high room with a steady temperature of $15^{\circ} \mathrm{C}$, no air circulation and the lights turned off. Hence, solar cell 1 is not able to harvest energy. However, the room temperature is low enough to recover energy via the TEG. During the first 20 minutes, the TEG was able to generate an average power of $107 \mu \mathrm{W}$, of which $37.5 \mu \mathrm{W}$ was effectively stored in the MEC, resulting in an overall system efficiency $\eta$ of $35.0 \%$. At the end of the first scenario, the test person stands up and walks to a switch for turning on the fluorescent lamps mounted at the ceiling. Then, he walks back to his original place and waits there in an upright position for the following 20 minutes. Solar cell 1 and the TEG are now both able to scavenge energy and generate, over the 20 minute interval, a total average power of $139.9 \mu \mathrm{W}$, of which $106.7 \mu \mathrm{W}$ is generated by the TEG and $33.2 \mu W$ by solar cell 1 . Eventually, 


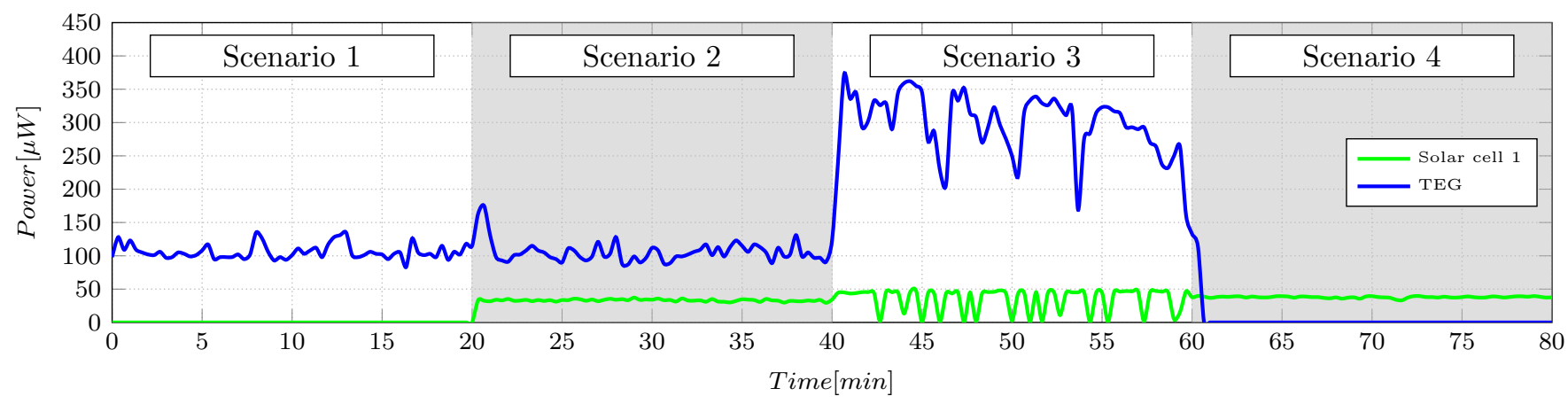

Fig. 4. Power generated by the TEG and solar cell 1 as a function of time in a realistic indoor environment.

$60.6 \mu \mathrm{W}$ was delivered on average to the MEC, leading to $\eta=43.2 \%$. Note that the peak in generated power by the TEG at the beginning of scenario 2 is caused by walking back and forth to the light switch. At the beginning of scenario 3, the test person starts walking and leaves the room, entering a $2.2 \mathrm{~m}$ high corridor with a steady temperature of $18^{\circ} \mathrm{C}$, no air circulation and similar fluorescent lights at the ceiling. During scenario 3, the test person keeps on walking, which results in a higher power generation by the TEG. However, at certain times, the wearer takes a break, resulting in drops in generated power by the TEG. The power generated by solar cell 1 also exhibits some drops. This is caused by the distance between subsequent lamps. On average, $34.8 \mu \mathrm{W}$ and $296.0 \mu \mathrm{W}$ was generated by solar cell 1 and the TEG, respectively. Eventually, an average power of $152.17 \mu \mathrm{W}$ was harvested in scenario 3, resulting in $\eta=46.0 \%$. At the end of scenario 3 , the test person returns to the same room as in scenario 1 and 2 and waits there in an upright position for the following twenty minutes. However, the room is now heated up to $25^{\circ} \mathrm{C}$ and the lights are turned on, which makes that only solar cell 1 is capable of scavenging energy. Specifically, solar cell 1 was able to generate $38.0 \mu \mathrm{W}$ on average, of which $25.0 \mu \mathrm{W}$ is effectively harvested and stored by the MEC. Fig. 4 clearly demonstrates the advantages of a hybrid energy harvesting approach. Including solar cell 1 in the design enables energy harvesting in the presence of artificial light (scenario 2 and 4), whereas including a TEG allows energy harvesting in the absence of light (scenario 1), provided ambient air temperature is low enough. If temperature is too high, the wearer could start walking in order to increase convection of the cold side of the TEG, since scenario 3 shows that walking increased power generation by the TEG. Second, scenarios 2 and 3 prove that, in certain situations, hybrid energy harvesting increases the total harvested energy by scavenging from two different energy sources. In general, wearing the proposed system in an outdoor environment will increase energy harvesting, thanks to improved light conditions and increased convection caused by wind. Moreover, in outdoor scenarios, solar cell 2 will also be able to harvest energy. [5] demonstrated that including solar cell 2 in the design allows scavenging an additional $49 \mathrm{~mW}$ in case solar cell 2 is placed perpendicular to the earth surface and pointed in the direction of the sun on a day with a turbidity-free sky.

\section{CONCLUSION}

In this contribution, we have described a compact and highly integrated wearable textile system, consisting of a wearable substrate integrated waveguide cavity-backed textile slot antenna on which two flexible solar cells, a flexible power management system and a MEC are integrated. In addition, a terminal for connecting an external TEG is provided. Measurements have demonstrated that the integration of the energy harvesting and power management hardware onto the selected wearable textile antenna has a negligible influence on its performance, proving that the selected topology is suitable as an integration platform. Furthermore, measurements in a real-life scenario were carried out to demonstrate that our system is able to combine the energy scavenged from artificial light in an indoor environment, from solar light in an outdoor environment and from the heat emanated by the user's body, in order to reduce the time, during which no energy is harvested, and to increase the total amount of harvested energy.

\section{REFERENCES}

[1] A. Dierck, S. Agneessens, F. Declercq, B. Spinnewyn, G.-J. Stockman, P. Van Torre, L. Vallozzi, D. Vande Ginste, J. Vanfleteren, T. Vervust, and H. Rogier, "Active textile antennas in professional garments for sensing, localisation and communication," International Journal of Microwave and Wireless Technologies, vol. 6, no. 3-4, pp. 331-341, 2014.

[2] M. A. Hanson, H. C. Powell Jr, A. T. Barth, K. Ringgenberg, B. H. Calhoun, J. H. Aylor, and J. Lach, "Body area sensor networks: Challenges and opportunities," Computer, vol. 42, no. 1, pp. 58-65, 2009.

[3] L. Huang, V. Pop, R. de Francisco, R. Vullers, G. Dolmans, H. de Groot, and K. Imamura, "Ultra low power wireless and energy harvesting technologies - an ideal combination," in Proc. IEEE Int. Conf. Commun. Syst., Singapore, 17-19 Nov. 2010, Conference Paper, pp. 295-300.

[4] P. Van Torre, L. Vallozzi, A. Dierck, H. Rogier, and M. Moeneclaey, "Power-efficient body-centric communications," in URSI Benelux Forum, 14 Sept. 2012, Conference Paper, pp. 8-10.

[5] S. Lemey, F. Declercq, and H. Rogier, "Textile antennas as hybrid energyharvesting platforms," Proc. IEEE, vol. 102, pp. 1833-1857, 2014.

[6] M. Lossec, B. Multon, H. Ahmed, and C. Goupil, "Thermoelectric generator placed on the human body: System modeling and energy conversion improvements," Eur. Phys. J, vol. 52, no. 1, pp. 1-10, 2010.

[7] V. Leonov, C. Van Hoof, and R. J. Vullers, "Thermoelectric and hybrid generators in wearable devices and clothes," in Proc. 6th Int. Workshop Wearable Implantable Body Sensor Netw., Berkeley, CA, USA, Jun. 3-5, 2009, Conference Paper, pp. 195-200.

[8] S. Lemey, F. Declercq, and H. Rogier, "Dual-band substrate integrated waveguide textile antenna with integrated solar harvester," IEEE Antennas Wireless Propag. Lett., vol. 13, no. 1, pp. 269-272, 2014.

[9] R. Moro, S. Agneessens, H. Rogier, and M. Bozzi, "Wearable textile antenna in substrate integrated waveguide technology," IET Electron. Lett., vol. 48, no. 16, pp. 985-987, 2012. 